\title{
Maladies de Tay-Sachs et de Sandhoff : les déficits en $\beta$-hexosaminidases, modèles de maladies des lysosomes
}

L'analyse moléculaire des déficits en $\beta$-hexosaminidases, prototype des maladies lysosomiales auxquelles appartiennent les maladies de Tay-Sachs et de Sandhoff, a été menée depuis plusieurs années par des méthodes variées qui permettent l'identification précise des lésions en cause. Ces travaux démontrent la grande hétérogénéité moléculaire de ces affections, y compris chez les Juifs Ashkénazes, et remettent en cause de façon définitive l'affirmation de leur confinement à cette ethnie. L'élaboration de thérapeutiques efficaces constitue aujourd'hui l'objectif majeur ; les recherches s'orientant dans deux directions principales, celle de la thérapie enzymatique et celle de la thérapie génique.

Jean-Claude Dreyfus Saïd Akli Livia Poenaru
ADRESSE

J.-C. Dreyfus : professeur honoraire au CHU Cochin-Port-Royal. S. Akli : étudiant en doctorat. L. Poenaru : professeur au CHU CochinPort-Royal. Institut Cochin de Génétique Moléculaire, Inserm U. 129, CHU Cochin, 24, rue du Faubourg-Saint-Jacques, 75014 Paris, France. es lysosomes sont des structures vésiculaires intracellulaires, chargées de la dégradation de substrats multiples. Ils contiennent au moins une quarantaine d'enzymes dont beaucoup sont sujettes à des déficits, cliniquement et biologiquement reconnaissables. Depuis dix ans, l'analyse moléculaire en a été entreprise, mettant en jeu des méthodes variées et complémentaires qui permettent de déterminer la nature de la lésion en cause, y compris l'analyse indépendante des deux allèles dans les cas apparemment homozygotes, en s'efforçant de corréler anomalies moléculaires et sévérité clinique. Ces travaux ont abouti au démembrement et à la classification des différentes formes de chaque maladie. Ils ont comme objectif ultime de conduire à des tentatives thérapeutiques qui s'avèrent particulièrement difficiles lorsque la maladie attaque le système nerveux central.

Dans cet article, nous décrirons brièvement les méthodes utilisées, valables dans tous les cas, en prenant pour exemple les déficits en $\beta$ hexosaminidases (HEX), qui sont parmi les mieux explorés. Ces déficits entraînent l'un des tableaux cliniques les plus impressionnants ; leur diagnostic prénatal, un des premiers à avoir été pratiqués, a abouti à leur quasi-disparition dans les ethnies les plus menacées. Leur analyse moléculaire, qui démontre plusieurs mécanismes connus, est sans doute la plus détaillée.

\section{Les hexosaminidases $[1,2]$}

Leur rôle principal dans la chaîne catabolique des gangliosides est de dégrader le ganglioside GM2 par 


\begin{abstract}
RÉFÉRENCES
1. Sandhoff K, Conzelmann E, Neufeld E, Kaback M, Suzuki K. The GM2 gangliosidoses. In : Scriver CR, Beaudet AL, Sly WS, Valle D, eds. The Metabolic Basis of Inherited Disease. New York : McGraw-Hill, 1989: 1807-39.

2. Neufeld EF. Natural history and inherited disorders of a lysosomal enzyme, $\beta$ hexosaminidase. J Biol Chem 1989; 264 : 10927-30.

3. Xie B, Wang W, Mahuran Don J. A Cys ${ }_{138}$-to-Arg substitution in the $G_{M 2}$ activator protein is associated with the $\mathrm{AB}$ variant form of $\mathrm{G}_{\mathrm{M} 2}$ gangliosidosis. $A m \mathrm{~J}$ Hum Genet 1992 ; 50 : 1046-52.

4. Myerowitz R, Hogikyan ND. A deletion involving Alu sequences in the $\beta$ hexosaminidase $\alpha$ chain gene of French Canadians with Tay-Sachs disease. J Biol Chem 1987 ; 262 : 15386-99.
\end{abstract}

5. Kaback M, Nathan T, Greenwald S. Tay-Sachs heterozygotes screening and prenatal diagnosis, USA experience and world prespectives. In : Kaback MM, ed. TaySachs Disease: Screening and Prevention. New York: AR Liss, 1977.

6. Triggs-Raine B, Akerman B, Clarke J, Gravel R. Sequence of DNA flanking the exons of the HEXA gene, and identification of mutations in Tay-Sachs disease. $A m J$ Hum Genet, 1991 ; 49 : 1041-54.

7. Akli S, Chelly J, Lacorte JM, Poenaru L, Kahn A. Seven novel Tay-Sachs mutations detected by chemical mismatch cleavage of PCR-amplified cDNA fragments. Genomics 1991 ; 11 : 124-34.

8. Akli S, Chomel JC, Lacorte JM, Bachner L, Kahn A, Poenaru L. Ten novel mutations in the HEX A gene in nonJewish Tay-Sachs patients. Hum Mol Genet 1992 (sous presse).

9. Mules EH, Hayflick S, Miller CS. Six novel deleterious and three neutral mutations in the gene encoding the $\alpha$ subunit of hexosaminidase $\mathrm{A}$ in non-Jewish individuals. Am J Hum Genet 1992 ; 50 : 834-41.

10. Navon R. Molecular and clinical heterogeneity of adult GM2 gangliosidosis.

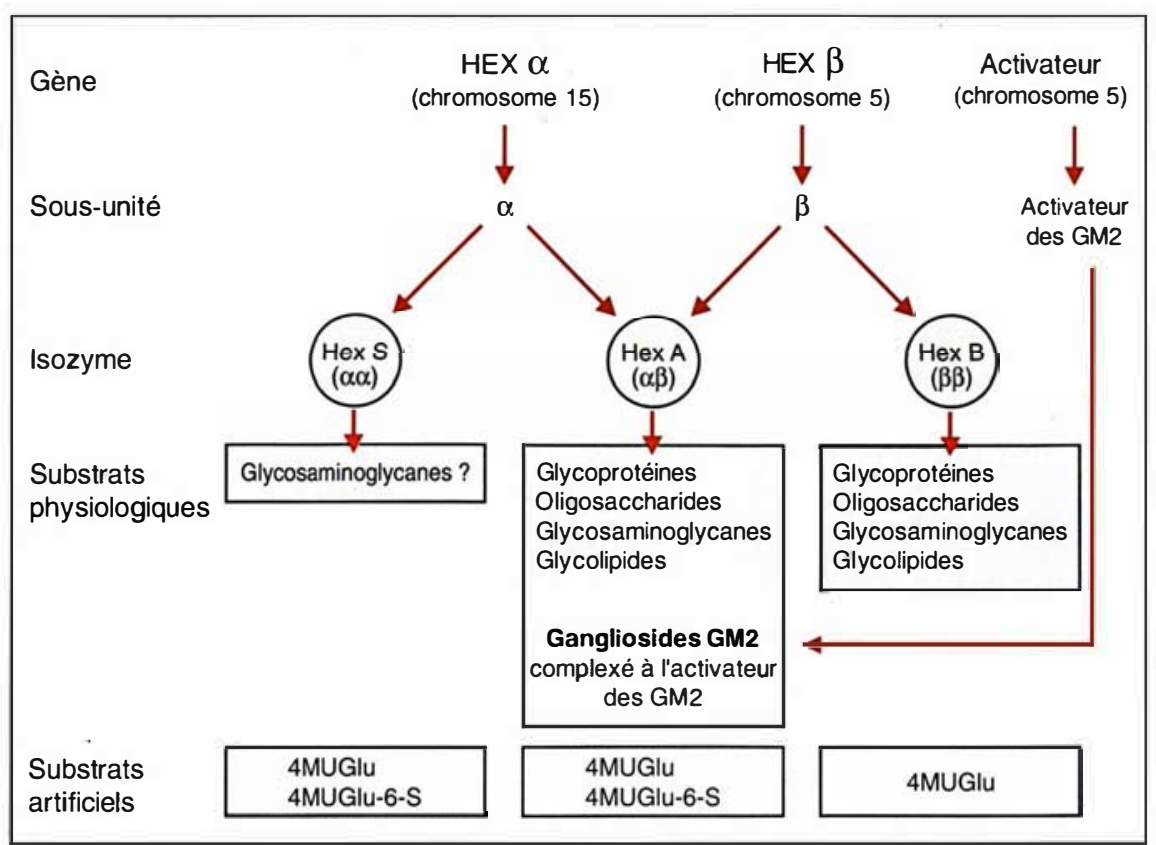

Figure 1. Substrats des hexosaminidases. $4 M U G l u=4$ méthylombelliféryl$\beta$-D-N-acétyl-glucosamine; $4 M U G l u-6-S=4$ méthylombelliféryl- $\beta$ - $D-N$-acétylglucosamine-6-sulfate (d'après [1]).

excision d'un groupement $\mathrm{N}$-acétylglucosamine ou galactosamine en configuration $\beta$. Elles sont formées de deux sous-unités : $\alpha$, dont le gène est porté par le chromosome 15 , et $\beta$, dont le gène est sur le 5 . Elles s'unissent pour former des dimères : $\alpha \beta$ forme l'HEX A, $\beta \beta$ forme l'HEX B. Seule HEX A est active sur le ganglioside GM2 ; pour fonctionner, elle réclame en outre la présence d'un activateur dont le gène est sur le chromosome 5. On connaît ainsi trois types de déficit : la maladie de TaySachs, où l'HEX A est déficiente à cause de l'absence de la sous-unité $\alpha$; la maladie de Sandhoff, où manque la sous-unité $\beta$; et un type $\mathrm{AB}$, où les deux sous-unités sont présentes mais l'activateur est absent. Nous ne reviendrons pas sur ce dernier type dont l'interprétation moléculaire en est à ses débuts. Une mutation dans le gène de l'activateur du GM2 vient d'être décrite [3].

Le diagnostic se fonde d'abord sur le dosage de l'enzyme. Si l'activité totale est effondrée dans la maladie de Sandhoff, où l'on ne trouve ni
l'HEX A ni l'HEX B, dans le TaySachs l'augmentation compensatrice de la sous-unité $\beta$ masque le déficit en HEX A secondaire à l'absence d' $\alpha$. Dans ce cas, l'électrophorèse sur acétate de cellulose permet de séparer l'HEX A de l'HEX B. On peut, en revanche, détecter les hétérozygotes par dosage différentiel de A (qui est thermolabile) et de B (thermostable), ou plus récemment par dosage spécifique de la A à l'aide d'un substrat sulfaté. Ce dosage est pratiqué, d'une part sur les familles des malades, d'autre part, systématiquement, dans les ethnies à risque. Certaines formes mutées (Tay-Sachs B1) agissent sur le substrat artificiel habituellement employé ; il faut dans ce cas s'adresser soit au substrat artificiel modifié (6-sulfaté), soit au substrat naturel (figure 1).

Les gènes des chaînes $\alpha$ et $\beta$, bien que portés par des chromosomes différents, se ressemblent. Ils ont une taille de 35-40 kb et sont constitués de 14 exons. $60 \%$ des nucléotides sont identiques et on pense qu'ils dérivent d'un ancêtre commun. $m / s n^{\circ} 8$, vol. 8, octobre 92 
Après la biosynthèse, chaque sousunité de l'HEX suit le sort habituel des enzymes destinées aux lysosomes : perte d'un peptide signal, passage dans le réticulum endoplasmique et le Golgi, N-glycosylation, acquisition du signal de reconnaissance mannose-6-phosphate, maturation protéolytique. La sous-unité $\alpha$ ne subit pas de maturation protéolytique et reste d'un seul tenant, tandis que $\beta$ est coupée en trois fragments, joints entre eux par des liaisons disulfure. Un élément essentiel est que les monomères restent inactifs, mais ils peuvent s'associer en dimères actifs $\alpha \beta$ (HEX A), $\beta \beta$ (HEX B) ou $\alpha \alpha$ (HEX S). Ce dernier, qui peut apparaître quand $B$ est déficient, est instable et catalytiquement très peu actif.

\section{Déficits}

\section{en hexosaminidases}

Le tableau clinique du déficit, qu'il s'agisse de la maladie de Tay-Sachs ou de celle de Sandhoff, est pratiquement le même. Il ne comporte pas d'anomalies morphologiques comme dans les mucopolysaccharidoses et l'aspect général n'est pas altéré. Mais dès la fin de la première année se produit un arrêt du développement psycho-moteur ; il apparaît des crises comitiales, une cécité accompagnée de la classique tache rouge cerise sur la macula de la rétine ; la mort survient au bout de quelques mois ou de quelques années, dans un tableau de retard psycho-moteur et intellectuel profond. Il existe toutefois des formes moins sévères, à début plus tardif (juvéniles, adultes) ou même asymp- tômatiques. Dans certains cas, des symptômes atypiques rendent le diagnostic délicat et, au moindre doute, il faut effectuer la recherche du déficit. Ces déficits peuvent être envisagés sous de multiples facettes : distinction entre les déficits en sousunités $\alpha$ et $\beta$, nature des lésions moléculaires, troubles de la maturation ou de l'assemblage, corrélations entre biologie moléculaire et gravité clinique, génétique des populations.

\section{Déficits en HEX A}

Ils sont les mieux connus et les plus fréquents. Pendant longtemps, on a vécu sur deux idées reçues : ils étaient l'apanage d'une seule ethnie, les Juifs Ashkénazes, jusqu'à ce qu'une fréquence aussi élevée (environ $3 \%$ de porteurs et un malade pour 2000 naissances) soit reconnue chez les Franco-Canadiens, puis que son existence, moins fréquente, soit constatée dans de nombreuses autres populations. L'autre notion $\left(\mathrm{m} / \mathrm{s} n^{\circ} 4\right.$, vol. 5, p. 267) voulait que, une seule ethnie étant le réservoir, la mutation devait provenir d'un effet fondateur et ne pouvait être qu'unique. Or, chez les Ashkénazes, existent deux mutations principales : la plus fréquente est une insertion de $4 \mathrm{pb}$ dans l'exon 11, créant un décalage de phase aboutissant à un codon stop 9 nucléotides en aval. L'autre mutation commune est un changement ponctuel, transversion $\mathrm{G} \rightarrow \mathrm{C}$ à la limite 5' de l'intron 12. L'épissage ne peut se faire et le taux d'ARNm est très bas. Une troisième forme, moins grave car se manifestant à l'âge adulte, remplace une Gly par une Ser au codon 269. La mutation commune des Franco-Canadiens [4] est une délétion de 7,6 kpb qui enlève le premier exon du gène $\alpha$; elle est probablement due à une recombinaison entre des séquences Alu. Elle représente 70 à $80 \%$ des déficits trouvés dans cette ethnie.

Dès nos premiers essais de diagnostic prénatal, il y a vingt ans, nous nous étions aperçus qu'en France la plupart des cas de Tay-Sachs diagnostiqués n'étaient pas d'origine ashkénaze. La quasi-ubiquité de cette notion s'est développée avec les succès remarquables de la campagne de dépistage des hétérozygotes [5] et de diagnostic prénatal, qui a fait pratiquement disparaître aux États-Unis et en Israël la naissance d'homozygotes chez les Ashkénazes, permettant d'orienter les projecteurs vers d'autres voies. L'analyse moléculaire a été rendue plus facile par l'emploi simultané de l'amplification de l'ADN, de l'électrophorèse sur gel des fragments amplifiés, en milieu non dénaturant ou en gradient de dénaturation (DGGE), des polymorphismes conformationnels simple brin, du clivage chimique des mésappariements (voir annexe 1). Le progrès le plus marquant a été la possibilité de partir de l'ARNm de fibroblastes, même lorsqu'il est en quantités minimes. Comme ces méthodes sont capables d'analyser séparément les deux allèles, elles révèlent si les malades sont des homozygotes ou des hétérozygotes composites.

On connaît aujourd'hui, pour le gène $H E X A$, environ 46 lésions moléculaires différentes [7, 8, 11, 12] (figure 2). Le plus grand nombre de mutations

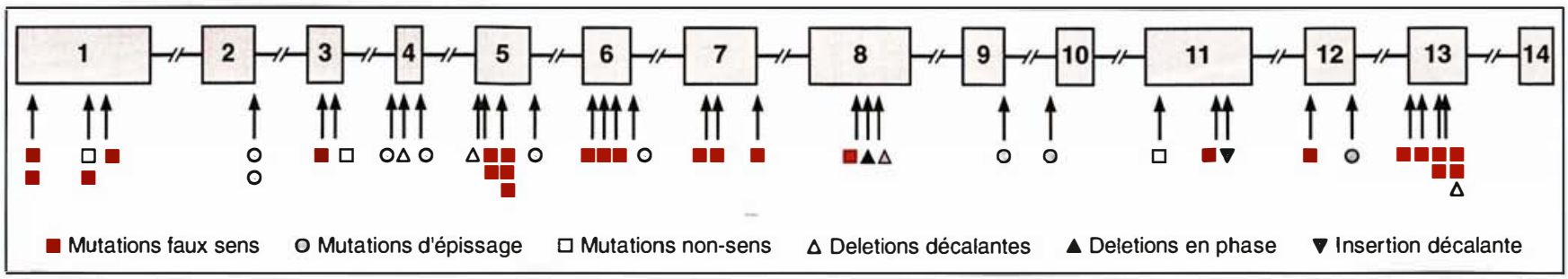

Figure 2. Mutations connues dans le gène de I'HEX A. 


\section{RÉFÉRENCES}

11. Akli S, Chelly J, Kahn A, Poenaru L. A null allele frequent in non-Jewish TaySachs patients. Hum Genet 1992 (sous presse).

12. Akli S, Chelly, Mézard C, Gandy S, Kahn A, Poenaru L.A "G " to "A " mutation at position-1 of a 5' splice site in a late infantile form of Tay-Sachs disease. $J$ Bio Chem $1990 ; 265$ : 7324-30.

13. Paw BH, Tieu PT, Kaback MM, Lim J, Neufeld EF. Frequency of three HEX A mutant alleles among Jewish and non-Jewish carriers identified in a Tay-Sachs screening program. Am J Hum Genet 1990 ; 47 : 698-705.

14. Neote K, McInnes B, Mahuran D, Gravel R. Structure and distribution of an Alu type deletion mutation in Sandhof disease. J Clin Invest 1990 ; 86 : 1524-31.

15. Nakano T, Suzuki K. Genetic cause of a juvenile form of Sandhoff disease. Abnormal splicing of $\beta$-hexosaminidase $\beta$-chain gene transcript due to a point mutation within intron 12. J Biol Chem 1989; 264 5155-8.

16. Dreyfus J, Poenaru L, Vibert $M$, Ravise N, Boue J. Characterization of a variant of $\beta$ hexosaminidase : "Hexosaminidase Paris ". Am J Hum Genet 1977 ; 29 : 287-93.

17. Dlott B, D’Azzo A, Quon D, Neufeld $\mathrm{E}$. Two mutations produce intron insertion in mRNA and elongated $\beta$-subunit of human $\beta$-hexosaminidase. J Biol Chem 1990 ; 265 : 17921-7.

18. Wakamatzu N, Kobayashi H, Miyatake T, Tsuti S. A novel exon mutation in the human $\beta$-hexosaminidase $\beta$-subunit gene affects 3' splice site selection $J$ Biol Chem 1992 ; 267 : 2406-13.

19. Ligtenberg MJL, Gennissen AMC, Vos HL Hilkens J. A single nucleotide polymorphism in an exon dictates allele dependent differential splicing of episialin mRNA. Nucleic Acids Res 1991; 19 : 297-301.

20. Kahn A, Briand P. Thérapie génique, espoirs et limites. médecine/sciences $1991 ; 7$ : 705-14. a été élucidé par deux groupes, celui de Gravel au Canada [6] et celui de L. Poenaru à Paris $[7,8]$, auxquelles viennent de s'ajouter six mutations nouvelles décrites par Mules et al [9]. Sans les décrire en détail, on peut les caractériser sous l'angle des mécanismes moléculaires, de la fréquence et de la distribution, de la gravité clinique et de l'homogénéité génétique [10].

- Dans la grande majorité des cas, sauf consanguinité, les deux allèles portent des mutations différentes, rendant d'autant plus difficile l'évaluation de la sévérité pour chaque allèle.

- Types de mutations. Les plus nombreuses sont les mutations fauxsens, un acide aminé étant remplacé par un autre. On a également trouvé des mutations d'épissage, des mutations non-sens, des mutations avec décalage de phase (telles qu'insertion de $4 \mathrm{pb}$ ou délétion de $2 \mathrm{pb}$ ) et une délétion d'un codon ( $\Delta$ Phe 304-305). Beaucoup de ces anomalies n'ont été retrouvées qu'une seule fois. Certaines sont plus fréquentes, comme la délétion Phe et surtout l'anomalie d'épissage de l'intron 9. Celle-ci conduit à l'activation d'un site donneur cryptique, à l'insertion de $17 \mathrm{pb}$ dans le transcrit et à une terminaison précoce (figure 3) [11]. Un intérêt particulier s'attache à une mutation d'épissage trouvée à l'état homozygote dans une seule famille [12]. Il s'agit d'une transition $\mathrm{G} \rightarrow \mathrm{A}$ de la dernière base de l'exon 5 ne changeant pas la séquence protéique, mais modifiant l'épissage de façon telle que seuls $3 \%$ des ARNm sont correctement épissés, alors que la grande majorité subit une délétion de l'exon 5 (figure 4, p. 802). Il en résulte une activité enzymatique résiduelle qui explique la forme atténuée de la maladie présente dans cette famille. - Récurrence des mutations dans la maladie de Tay-Sachs. Cette question est importante en génétique des populations : si l'on trouve la même mutation en plusieurs endroits du globe, cela est-il dû à des migrations, des filiations méconnues ou à des récurrences des mêmes lésions moléculaires? La mutation la plus fréquente chez les Ashkénazes, l'insertion de $4 \mathrm{pb}$ dans l'exon 11 , a été retrouvée, à l'état homozygote ou hétérozygote, par plusieurs auteurs dans des familles en principe non juives, notamment en France, au Canada et aux États-Unis. Dans une étude panethnique [13], la fréquence de cet allèle était de $73 \%$ chez les Juifs et de $16 \%$ chez les non-Juifs. L'explication la plus simple est qu'il existait en Europe un ancêtre commun porteur de cette mutation. Cette explication peut aussi être invoquée pour la forme adulte Gly $269 \rightarrow$ Ser, trouvée avec des fréquences voisines dans diverses populations. En revanche, elle s'accorde mal avec l'observation de la mutation d'épissage de l'intron 12, qui n'est pas retrouvée du tout dans les autres ethnies. Il existe donc probablement des mutations récurrentes, mais, pour l'affirmer, il serait nécessaire de connaître les haplotypes des malades, c'est-àdire l'enchaînement de plusieurs marqueurs polymorphiques de l'ADN, voisins du gène $H E X A$, permettant de déterminer si deux mutations similaires sont portées par des fragments d'ADN provenant d'un ancêtre commun ou de deux ancêtres différents. Il en va de même pour les autres mutations trouvées plusieurs fois.

\section{Déficits en HEX B}

Le déficit complet en HEX B provoque la maladie de Sandhoff, très peu différente, nous l'avons dit, de la maladie de Tay-Sachs sur le plan clinique. Le diagnostic biologique en est facile, en raison de l'effondrement de l'activité de l'HEX totale. Comme dans le Tay-Sachs, à côté de la forme infantile dévastatrice, il existe des formes retardées et même des déficits asymptomatiques. La fréquence est beaucoup plus faible que celle du Tay-Sachs. Aucune ethnie ne la concentre dans son ensemble, mais on a décrit des zones localisées où la concentration est forte, comme dans le Saskatchewan canadien chez des métis d'Indiens et de FrancoCanadiens, ou en Argentine dans la région de Córdoba [14].

Les recherches moléculaires ont été peu nombreuses. La forme la plus fréquente (plus de $25 \%$ des cas) est due à une délétion englobant le promoteur et les cinq premiers exons, par recombinaison entre des séquences Alu. Retrouvée par Neote et 
al. [14] dans des lignées provenant de dix ethnies différentes, elle pourrait dériver d'une origine commune ; mais il est au moins aussi probable qu'elle se soit produite plusieurs fois, connaissant la fréquence des recombinaisons Alu-Alu. Une autre mutation retrouvée plusieurs fois est due à la présence d'un site d'épissage supplémentaire dans l'intron 12, provoquant une insertion de huit acides aminés [15]. Une mutation responsable de la synthèse d'une HEX B allongée (de six acides aminés) représente la lésion moléculaire d'une forme inapparente qui a été très étudiée (HEX Paris [16]). Une duplication d'une zone de jonction entre l'intron 13 et l'exon 14 crée un site d'épissage alternatif insérant 18 bases dans l'ARNm. A cet épissage anormal échappe une partie de l'ARN, expliquant l'activité résiduelle et la bénignité clinique [17]. Enfin, des auteurs japonais [18], ont décrit une forme juvénile dans laquelle une mutation exonique ponctuelle active un site cryptique d'épissage, éliminant la plus grande partie de l'exon 11. Bien que déjà décrite, notamment pour le gène de l'épisialine [19], une telle mutation ponctuelle, non située au voisinage immédiat d'une jonction (en position 8 de l'exon) n'est pas très habituelle dans les anomalies d'épissage.

\section{Traitement}

Il n'existe, à l'heure actuelle, aucun traitement des déficits en hexosaminidase, ce qui justifie la poursuite du diagnostic prénatal. Pour l'avenir, deux voies se partagent les espoirs. - L'apport direct d'enzyme à l'intérieur des cellules. Cette méthode a obtenu des succès incontestables dans la maladie de Gaucher due au défi- cit en une $\beta$-glucosidase $\left(\mathrm{m} / \mathrm{s} n^{\circ} 7\right.$, vol. 7, p. 752), et peut en espérer d'autres, surtout lorsque le système nerveux est peu ou pas en cause. Lorsqu'il l'est, il reste à vaincre les problèmes de perméabilité (barrière hémato-encéphalique, accès intracellulaire aux lysosomes). Des résultats encourageants ont été récemment enregistrés $\left(\mathrm{m} / \mathrm{s} n^{\circ}\right.$ 5, vol. 8, p. 499). - La thérapie génique. Il est facilement concevable d'insérer un ADNc de moins de $2 \mathrm{~kb}$ dans un vecteur. Une étape essentielle des essais réclame la possession de modèles animaux. On connaît, chez le chat, un déficit en HEX B. Pour HEX A, ou plutôt la sous-unité $\alpha$, des travaux sont en cours pour créer des modèles par recombinaison homologue, mais aucun résultat positif n'a encore été publié.

Les études moléculaires des déficits en hexosaminidases ont permis de

HEX A normal

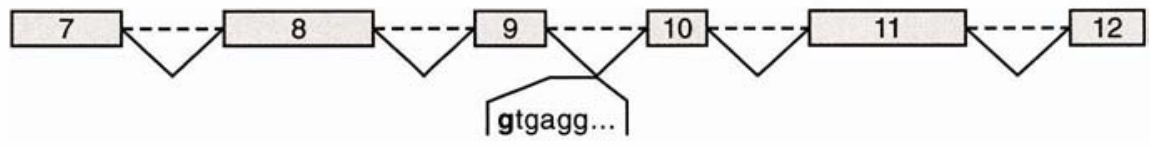

ARNm normal

\begin{tabular}{|l|l|l|l|l|l|}
\hline 7 & 8 & 9 & 10 & 11 & 12 \\
\hline
\end{tabular}

HEX A mutant

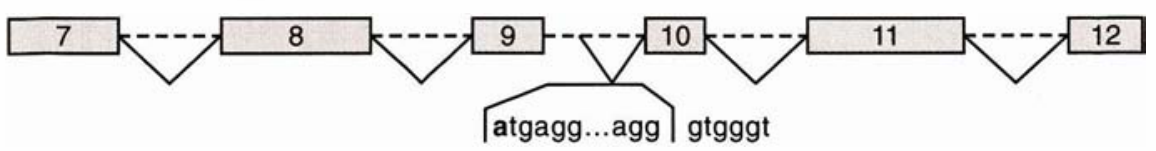

$17 n t$

ARNm mutant avec insertion de 17 bases

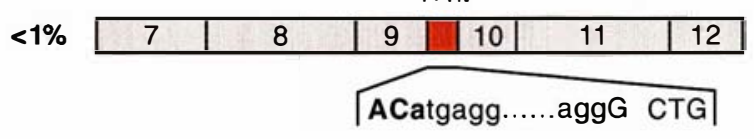

$<1 \%$

ACatgagg.......aggG CTG

Figure 3. Représentation schématique des produits de l'excision-épissage normal ou aberrant de I'ARN précurseur HEX A dans le cas d'une mutation intronique (mutation GT $\rightarrow$ AT au site donneur d'épissage de I'intron 9). Cette mutation abolit l'épissage au site donneur normal et moins de $1 \%$ des transcrits utilisent un site cryptique activé $17 p b$ en aval. L'utilisation de ce site induit l'insertion des $17 p b$ dans le transcrit conduisant à un décalage de phase de lecture et à l'apparition d'un codon stop prématuré. 


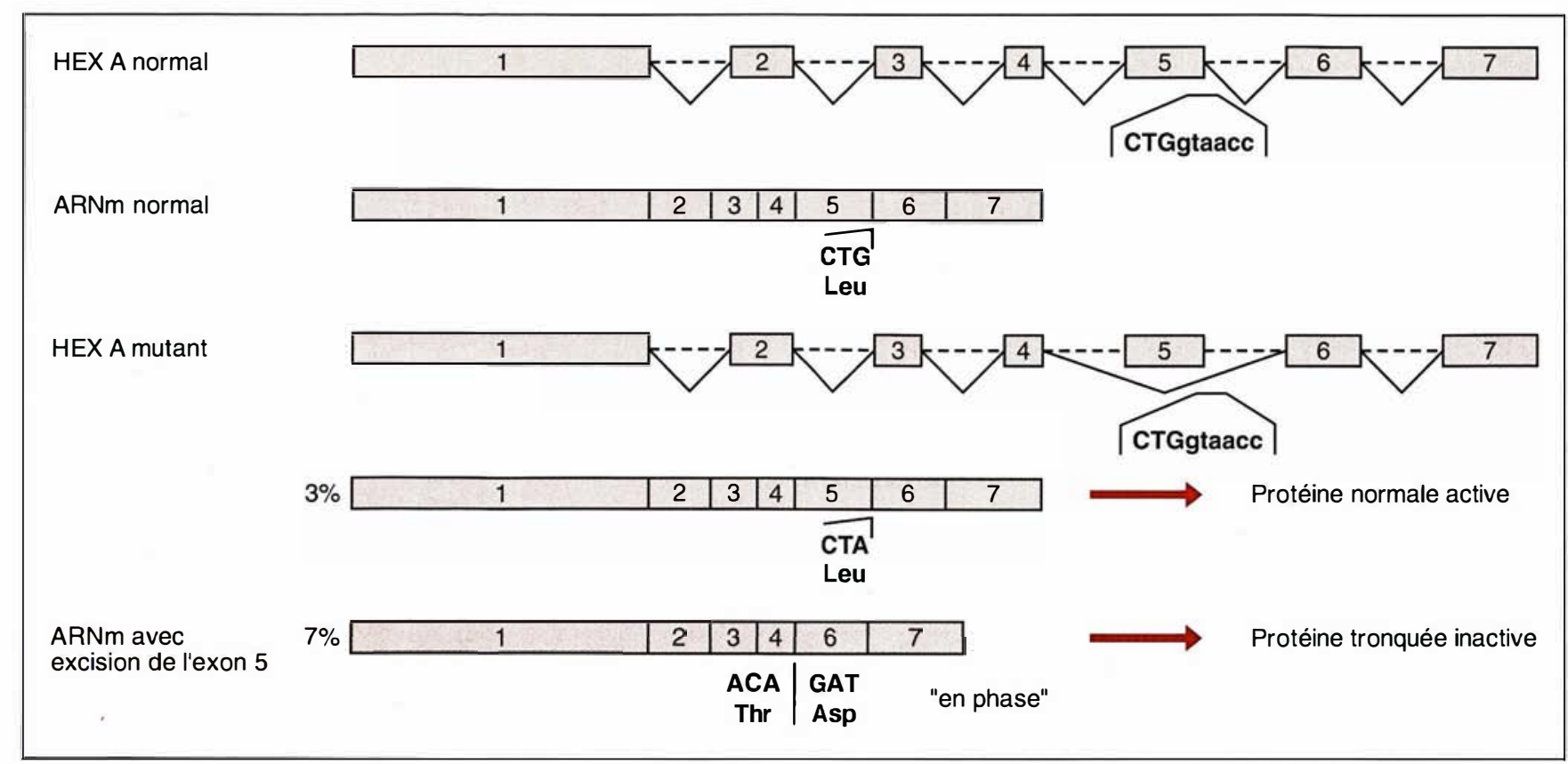

Figure 4. Représentation schématique des produits de l'excision-épissage normal ou aberrant de I'ARN précurseur HEX A dans le cas d'une mutation exonique (mutation $\rightarrow$ A de la dernière base de l'exon 5 ) qui diminue l'efficacité de l'épissage. $3 \%$ des transcrits sont correctement épissés et conduisent à la synthèse d'une protéine normale puisque la base mutée ne modifie pas le sens du codon et $97 \%$ des transcrits ont subi l'excision de l'exon 5, conduisant probablement à la synthèse d'une protéine tronquée inactive.

conclure quant à la grande hétérogénéité moléculaire de ces affections. Cela s'oppose à envisager leur diagnostic par étude directe de l'ADN ou de l'ARN. Cependant, devant l'observation d'un déficit enzymatique, on peut, grâce à cette approche, distinguer les formes adultes des formes infantiles classiques, ou des formes à phénotype particulier.

$\mathrm{Si}$, actuellement, les méthodes de diagnostic sont tout à fait maîtrisées, l'absence de toute thérapie efficace incite à un développement scientifique dans ce sens. Les perspectives d'une thérapie génique permettant le transfert in vivo d'ADN thérapeutique sont peut-être lointaines, mais méritent d'être explorées. Dans le cas de ces affections, les difficultés résident en la nécessité d'un traitement extrêmement précoce, prénatal dans les formes infantiles habituelles, et d'une correction des neurones protégés par la barrière hémato-encéphalique. La mise au point de vecteurs efficaces et inoffensifs, adénoviraux [20] ou autres, capables de véhiculer le transgène dans le système nerveux central est ainsi une priorité pour les équipes engagées dans cette voie de recherche, dont la nôtre

\section{Summary}

Tay-Sachs and Sandhoff's diseases : deficiencies in $\beta$-hexosaminidases, a model for lysosomal diseases

The deficiency in $\beta$-hexosaminidases is the prototype of lysosomal diseases. The knowledge of molecular mechanisms involved has greatly progressed since a few years, thanks to the use of new techniques, the principle of which is analyzed here. The genes and their products, $\alpha$ and $\beta$ chains, are briefly described. The main emphasis is placed on their abnormalities. $\alpha$ chain disorders, responsible for Tay-Sachs disease, have been the most studied. More than 40 different molecular lesions are known: they are no more confined to Ashkenazi Jews as it was believed. All types of mutations have been found, some being recurrent in various countries, and most of the apparent homozygotes are in fact compound heterozygotes. $\beta$ chain deficiencies, resulting in Sandhoff's disease, have been less studied; deletions, insertions and splicing alterations have been described. Therapeutic attempts are not yet successful, and the only alternative is antenatal diagnosis. Consequently, among Ashkenazi Jews in USA and in Israel the birth of affected homozygotes has been nearly suppressed. For the future great hopes are placed in gene therapy.

TIRÉS A PART

J.-C. Dreyfus. 


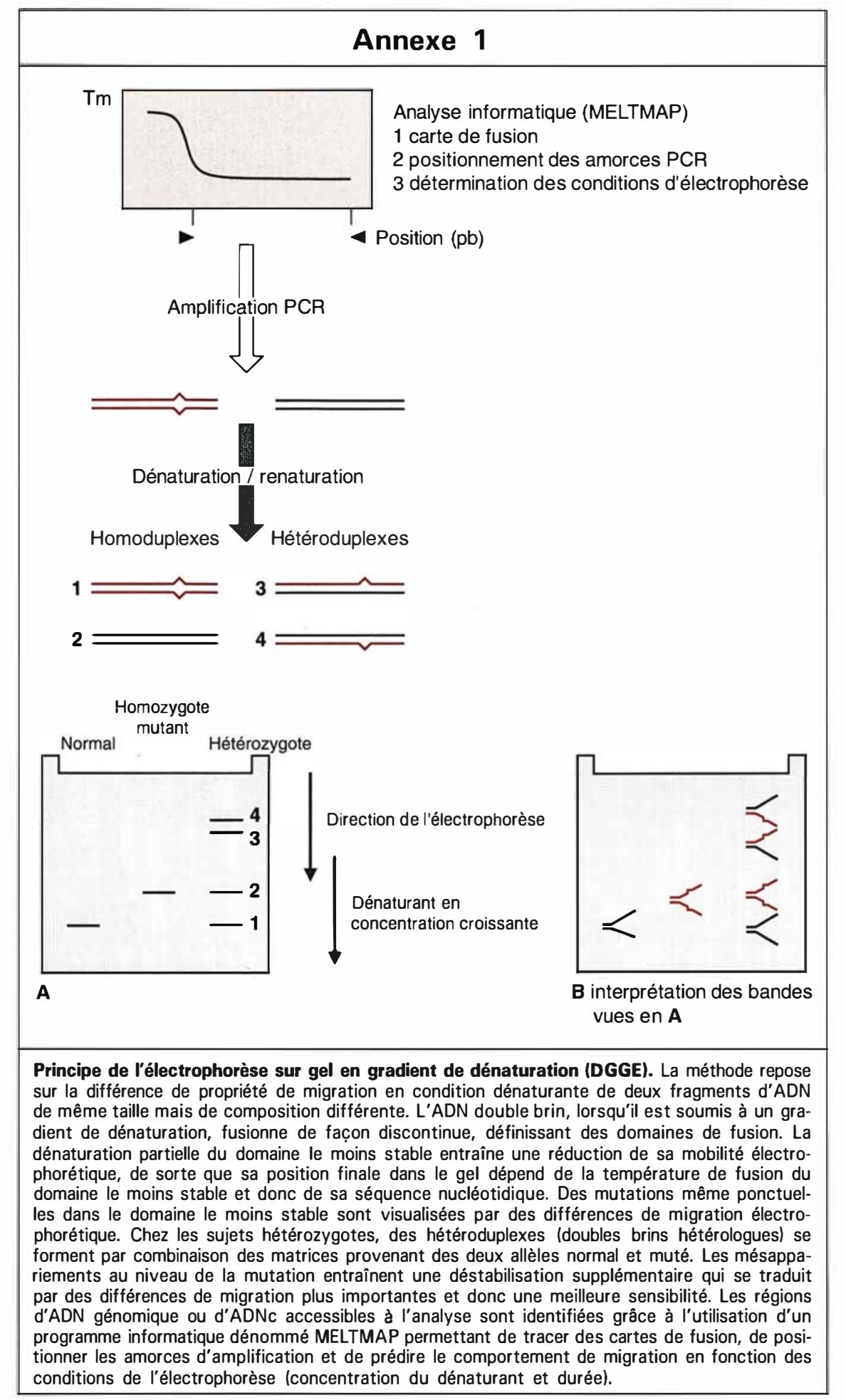

\section{Annexe 2}

Polymorphisme de conformation simple brin (SSCP). Le principe de cette méthode est fondé sur le fait que l'ADN simple brin adopte, au cours de sa renaturation, une conformation particulière dépendant de sa séquence nucléotidique et influençant sa mobilité électrophorétique. Les produits d'amplification radiomarqués sont dénaturés puis soumis à une électrophorèse de haute résolution en gel de polyacrylamide non dénaturant. Une différence de séquence d'une seule base entre l'ADN à analyser et l'ADN de référence sera visualisée par un décalage de migration. 


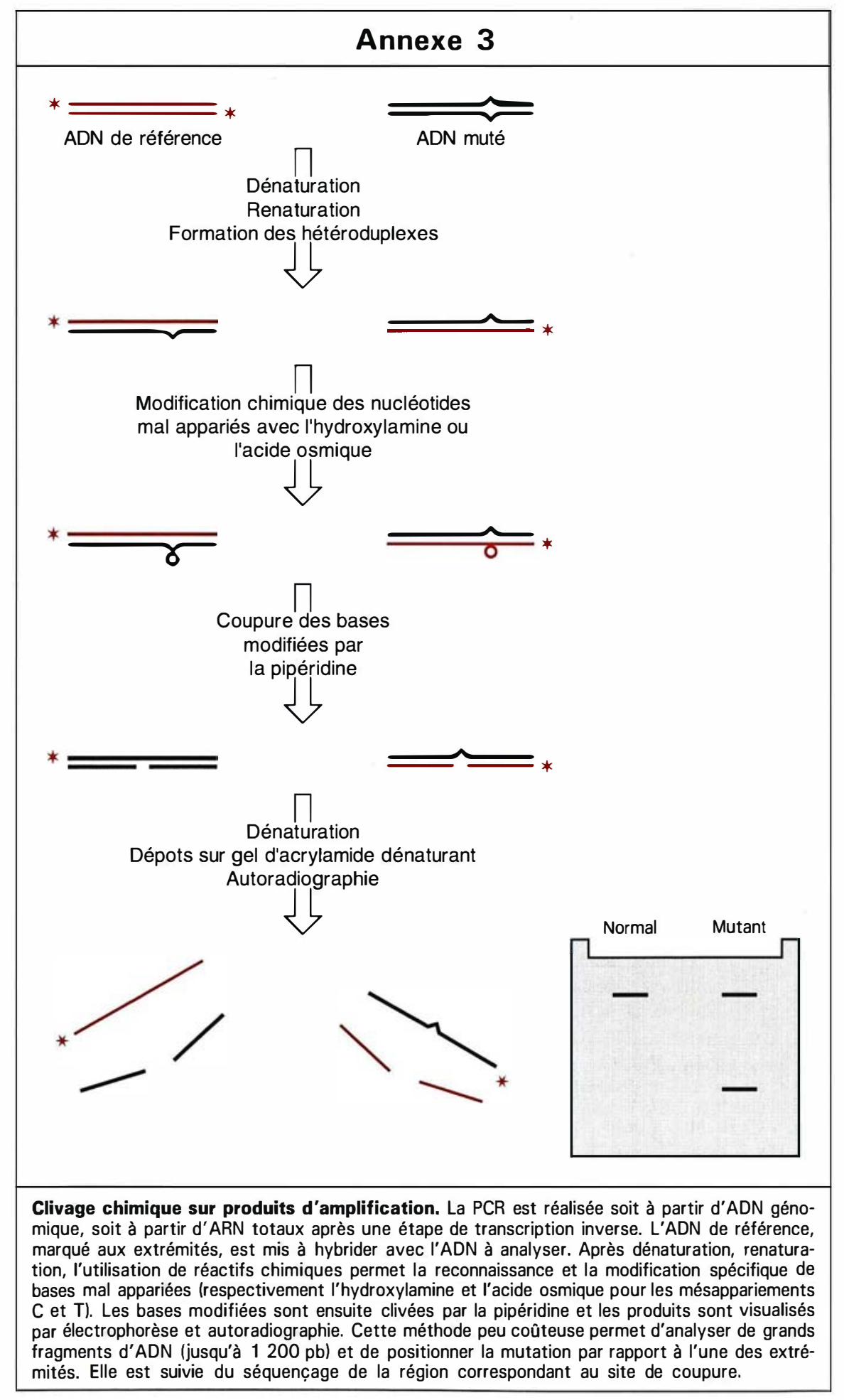

